\title{
Fibronectin 1 protein expression in clear cell renal cell carcinoma
}

\author{
S. STEFFENS ${ }^{1 *}$, A.J. SCHRADER ${ }^{3 *}$, GESA VETTER $^{1}$, H. EGGERS $^{1}$, H. BLASIG $^{1}$, \\ J. BECKER ${ }^{2}$, M.A. KUCZYK ${ }^{1}$ and J. SERTH ${ }^{1}$ \\ Departments of ${ }^{1}$ Urology, and ${ }^{2}$ Pathology, Hannover Medical School, Hannover; \\ ${ }^{3}$ Department of Urology, Ulm Medical School, Ulm, Germany
}

Received September 23, 2011; Accepted December 12, 2011

DOI: 10.3892/ol.2012.566

\begin{abstract}
Fibronectin 1 (FN1) is a glycoprotein that is involved in cell adhesion and migration processes including embryogenesis, wound healing, blood coagulation, host defenses and metastasis. The aim of this study was to elucidate the FN1 protein expression in renal cell carcinoma (RCC) and to determine its potential prognostic relevance. A total of 270 clear cell RCC tissue specimens were collected from patients undergoing surgery for renal tumors. Biomarker expression was determined by immunohistochemistry and correlated with clinical variables. Survival analysis was carried out for 153 patients with complete follow-up data and pathologically proven clear cell carcinoma of the kidney. The follow-up group had a mean follow-up period of 83.8 months (IQR 26.2-136.2 months). The calculated median 5-year overall and tumor-specific survival rate of all 153 evaluable patients was 66.6 and $71.0 \%$, respectively. A higher diseaserelated mortality rate was observed among patients with cytoplasmic FN1 expression (41.3 vs. 24.7\%, p=0.039, Fisher's exact test). No significant correlation was found between FN1 staining and patient characteristics such as age, gender, tumor differentiation and visceral metastasis. However, there was a trend for $F N 1$ expression and correlation with tumor stage and lymph node metastasis ( $\mathrm{p}=0.085$ and $\mathrm{p}=0.203$; respectively). The Kaplan-Meier analysis revealed significant differences in the 5-year tumor-specific survival for patients with and without cytoplasmic FN1 expression (64.8 vs. $77.7 \%$; $\mathrm{p}=0.035$, log-rank test). However, results of the multivariate Cox regression analysis showed that FN1 expression was not an independent marker of either overall or tumor-specific survival. In conclusion, FN1 protein expression in RCC is associated with a higher disease-related mortality rate, indicating a possible role
\end{abstract}

Correspondence to: Dr Markus A. Kuczyk, Department of Urology, Hannover Medical School, Carl-Neuberg-Str. 1, D-30625 Hannover, Germany

E-mail: makuczyk@gmx.de

${ }^{*}$ Contributed equally

Key words: renal cell carcinoma, fibronectin 1, prognosis in RCC progression. Therefore, our data on FN1 encourage further investigations to determine the role of FN1 in RCC.

\section{Introduction}

Renal cell carcinoma (RCC) is a common urological tumor and accounts for approximately $3 \%$ of all human malignancies (1). A significant increase in its incidence has been observed during the last few decades, and the annual mortality-to-incidence ratio for RCC is considerably higher than for other tumors of the genitourinary tract (1). RCC encompasses numerous histological subtypes with distinct genetic and biological features that determine clinical course and outcome (2). Tumor characteristics such as tumor stage and grade appear to have limited value in predicting the clinical outcome of individual patients, as approximately $50 \%$ of patients who undergo surgery with curative intent for less advanced disease are expected to develop a distant recurrence. Therefore, an increased understanding of genetic and biological changes could aid in the development of a valuable marker to improve the individual therapeutic management and clinical outcome of RCC.

An essential step in the formation of metastases and in local disease progression is the invasion of tumor cells in the extracellular matrix. Cell adhesion molecules and extracellular matrix proteins support either an increase or a decrease in the ability of tumor cells to adhere to surrounding tissue. Among the few extracellular matrix proteins identified, fibronecin (FN) appears to play a significant role in the inhibition and promotion of cellular attachment by interacting with various receptors.

FN is a glycoprotein that is involved in cellular adhesion and migration processes including embryogenesis, wound healing, blood coagulation, host defenses and metastasis. The molecule is widely distributed in healthy membranes, lamina propria, vessel structures, nerves and smooth-muscle cell layers (3). However, the function of FN is not clearly known $(4,5)$.

At present, no specific tumor markers for the differential diagnosis of RCC are available. Previously, we showed that fibronectin 1 (FN1) mRNA expression in RCC is significantly higher compared to that in normal renal tissue (6). Furthermore, patients with advanced disease exhibited significantly higher FN1 expression compared to localized disease, indicating a possible role in renal carcinogenesis (6). Therefore, the aim of this study was to elucidate the protein expression of FN1 
in $\mathrm{RCC}$ and to determine a possible prognostic relevance for optimal clinical management.

\section{Materials and methods}

Tissue specimens. The present study included 270 patients who underwent radical nephrectomy between 1979 and 1998. Tissue was obtained from archival routine surgical specimens. The tissue samples were selected by a pathologist and prepared from the primary tumor and arranged on tissue microarrays (TMA) as described previously (12). Two pathologists evaluated the specimens with respect to tumor stage, grade and histological subtypes. Tumor samples were classified primarily according to the UICC 1997 TNM tumor staging system and nuclear grading was based on the Fuhrman grading system (7). At the time of the pathological assessment of our specimens, the UICC 2002 version was not available. Histological subtypes were assessed according to the consensus classification of renal cell neoplasia (8). Data were collected by physicians and data managers and subsequently maintained using a relational database. The study was approved by the ethics committee of the institution.

Patients. The median age of the cohort was 60.3 years $(\mathrm{SD} \pm$ 11.2 months). A total of 155 patients were male (57.4\%), and 115 patients were female (42.6\%). In total, 7, 143, 107 and 13 patients presented with pT1, pT2, pT3 and pT4, respectively. Tumor differentiation showed that 43,167 and 19 patients suffered from G1, G2 and G3/4 tumors, respectively. Furthermore, 27 patients presented with lymph nodes and 54 patients with visceral metastasis.

In the majority of cases, data regarding the cancer-specific long-term survival (CSS) were retrieved from electronic patient charts. The duration of the follow-up was calculated from the date of surgery to the date of mortality or last follow-up. Mortality was assessed as either cancer-related or unrelated. Survival analysis was carried out for 153 patients with complete follow-up data and pathologically proven clear cell carcinoma of the kidney. The follow-up group exhibited a mean follow-up period of 83.8 months [interquartile range (IQR) 26.2-136.2 months]. At the time of the last follow-up examination, 80 patients were alive, 51 patients had succumbed to progressive RCC and 22 patients to other causes.

Procedures. Expression of FN1 was determined by immunohistochemistry (IHC). The paraffin-embedded TMA samples were deparaffinized, rehydrated and immersed in 3\% hydrogen peroxide solution to block endogenous peroxidase activity. Antigen retrieval was accomplished by microwave heating specimens in a $0.01 \mathrm{M}$ citrate buffer for $15 \mathrm{~min}$. Biomarker expression was immunohistochemically detected by commercially available antibodies (FN1 rabbit polyclonal antifibronectin-1, Becton-Dickinson Biosciences, Franklin Lakes, NJ, USA). The optimal dilution was 1:100. Following $12 \mathrm{~h}$ of incubation the sections were washed in TBS and incubated with a secondary biotinylated antibody (Vectastatin Elite ABC Kit, Vecto Laboratories, Inc., Burlingame, CA, USA) for $60 \mathrm{~min}$. The DAB system (Vector) was used for visualization according to the manufacturer's instructions. Sections were briefly rinsed in tap water, counterstained with Mayer's hematoxylin solu- tion and then mounted. For negative controls, the primary antibody was replaced by non-immune serum. Tissue staining was assessed in a blind study by two independent investigators (H.B and S.S.). The expression of FN1 was evaluated in the membrane, cytoplasm and nucleus of the tumor cells. The staining reaction was classified according to a semi-quantitative IHC reference scale, as previously described (9-13).

Statistical analysis. Follow-up duration was calculated from the date of surgery to the date of mortality or the last follow-up visit. The primary endpoints of this study were tumor-specific and overall survival. Continuous variables were reported as the means and standard deviations (SD) for parametric distributions or as medians and IQRs for non-parametric distributions. Kaplan-Meier survival times were calculated, and subgroups were compared by the log-rank test. Multivariate Cox regression models were used to assess the association between survival and cell membranes as well as cytoplasmic staining adjusted for various clinical and patient covariates. The Chi-square and Fisher's exact tests were conducted to assess associations between cell membrane/cytoplasm and patient/tumor-specific characteristics. SPSS 17.0 was used for statistical assessment. $\mathrm{P}<0.05$ was considered to indicate a statistically significant difference in all tests. All p-values were two-sided.

\section{Results}

Correlation between patient characteristics and FN1 expression. No significant correlation was found between FN1 staining in the cytoplasm and patient chraracteristics such as age, gender, tumor differentiation and visceral metastasis. However, there was a trend for FN1 expression and correlation with tumor stage and lymph node metastasis $(\mathrm{p}=0.085$ and $\mathrm{p}=0.203$; respectively; Table I). Cell nuclei were generally FN1-negative in the tumor specimens.

FN1 expression and clinical course. The calculated median 5 -year overall and tumor-specific survival rate of all 153 evaluable patients was 66.6 and $71.0 \%$, respectively.

With a median follow-up of 83.8 months (IQR 26.2-136.2 months), a higher disease-related mortality rate was observed among patients with cytoplasmic FN1 expression ( 41.3 vs. $24.7 \%$, p=0.039, Fisher's exact test). The Kaplan-Meier analysis revealed significant differences in 5 -year tumor-specific survival for patients with and without cytoplasmic FN1 expression (64.8 vs. $77.7 \%$; p =0.035, log-rank test; Fig. 1).

Multivariate Cox regression analysis was conducted to analyze the correlation of FN1 expression and clinicopathological characteristics including age, gender, stage, metastatic status and tumor grade. The results showed that FN1 expression was not an independent marker of either overall or tumor-specific survival (Table II).

\section{Discussion}

FN and its receptors are crucial in mediating cell adhesion, migration and signal transduction, and possibly limiting the prevention of apoptosis in certain tissues (14). It is a component of the extracellular matrix, and cells adhere to $F N$ via 
Table I. Association of different patient and tumor specific characteristics with FN1 expression in tumor cell cytoplasm.

\begin{tabular}{|c|c|c|c|c|}
\hline Characteristics & FN1 staining negative & FN1 staining positive & P-value & Test \\
\hline Age $($ mean $\pm \mathrm{SD})$ & $61.2 \pm 11.8$ years & $59.3 \pm 10.6$ years & 0.17 & T-test \\
\hline Gender & & & 0.14 & Fisher's exact \\
\hline Female & $63(47.4 \%)$ & $52(38.0 \%)$ & & \\
\hline Male & $70(52.6 \%)$ & $85(62.0 \%)$ & & \\
\hline Stage (TNM 2002) & & & 0.09 & $\chi^{2}$ \\
\hline pT1 & $5(3.8 \%)$ & $2(1.5 \%)$ & & \\
\hline pT2 & $78(58.6 \%)$ & $65(47.4 \%)$ & & \\
\hline pT3 & $43(32.3 \%)$ & $64(46.7 \%)$ & & \\
\hline pT4 & $7(5.3 \%)$ & $6(4.4 \%)$ & & \\
\hline LN metastasis ${ }^{\mathrm{a}}$ & & & 0.20 & Fisher's exact \\
\hline $\mathrm{pN}-$ & $64(88.9 \%)$ & $80(80.8 \%)$ & & \\
\hline $\mathrm{pN}+$ & $8(11.1 \%)$ & $19(19.2 \%)$ & & \\
\hline Pulmonary/visceral metastasis ${ }^{\mathrm{a}}$ & & & 0.88 & Fisher's exact \\
\hline M- & $99(79.2 \%)$ & $101(78.3 \%)$ & & \\
\hline $\mathrm{M}+$ & $26(20.8 \%)$ & $28(21.7 \%)$ & & \\
\hline Grade & & & 0.30 & $\chi^{2}$ \\
\hline G1 & $25(21.6 \%)$ & $18(15.9 \%)$ & & \\
\hline $\mathrm{G} 2$ & $84(72.4 \%)$ & $83(73.5 \%)$ & & \\
\hline $\mathrm{G} 3 / 4$ & $7(6.0 \%)$ & $12(10.6 \%)$ & & \\
\hline
\end{tabular}

${ }^{\text {aAt }}$ time of renal surgery. FN1, fibronectin 1; SD, standard deviation; LN, lymph node.
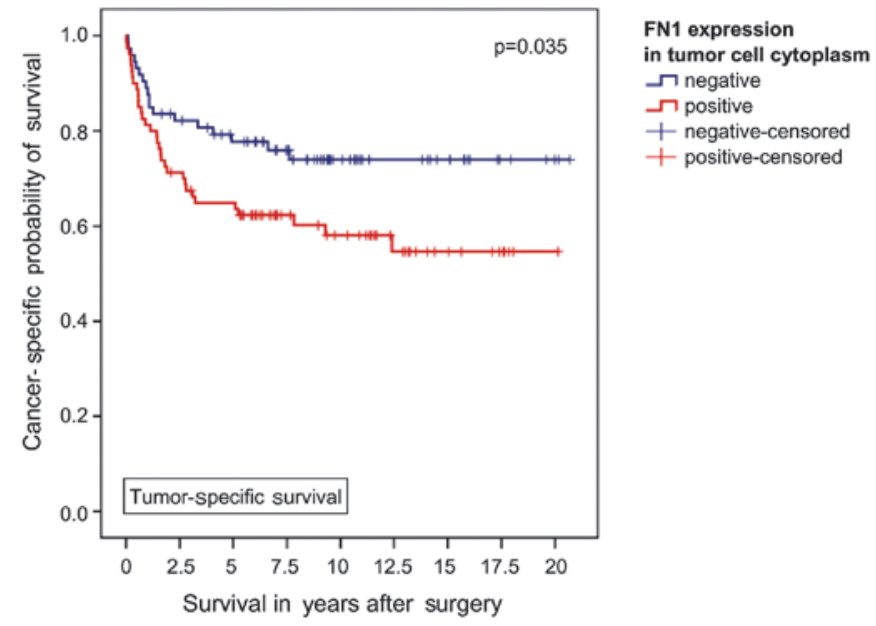

Figure 1. Association between FN1 expression and clinical outcome in all patients (Kaplan-Meier; $n=153$ evaluable): The tumor-specific survival of patients with FN1 expression was significantly shorter; 5-year tumor-specific survival rates were calculated at 64.8 and $77.7 \%(\mathrm{p}=0.035$, Mantel-Cox)

the integrin transmembrane receptors. Stimulation of integrins and growth factor receptors is required to enhance cell cycle progression through the G1 phase, and mitogenic signaling is weak and transient in the absence of integrin-mediated cell adhesion (15). Using IHC, we showed that a higher diseaserelated mortality rate was observed among patients with cytoplasmic FN1 expression. This novel finding in a human tumor specimen supports the suggestion that FN1 may play
Table II. Multivariate Cox regression analysis of FN1 expression and clinicopathological characteristics. ${ }^{\mathrm{a}}$

\begin{tabular}{|c|c|c|}
\hline Characteristics & HR (95\% CI) & P-value \\
\hline Age & $1.003(0.97-1.03)$ & 0.85 \\
\hline Gender & & 0.12 \\
\hline Female & 1 (Reference) & \\
\hline Male & $1.77(0.86-3.61)$ & \\
\hline T-stage & & 0.22 \\
\hline pT1 & 1 (Reference) & \\
\hline pT2 & $1.04(0.14-8.01)$ & 0.97 \\
\hline pT3 & $1.77(0.23-13.78)$ & 0.59 \\
\hline pT4 & $3.73(0.33-42.65)$ & 0.29 \\
\hline Metastatic disease & & $<0.001$ \\
\hline N/M- & 1 (Reference) & \\
\hline $\mathrm{N} / \mathrm{M}+$ & $5.82(2.95-11.45)$ & \\
\hline Differentiation & & 0.003 \\
\hline G1 & 1 (Reference) & \\
\hline G2 & $3.32(0.99-11.12)$ & 0.51 \\
\hline $\mathrm{G} 3 / 4$ & $10.92(2.58-46.14)$ & 0.001 \\
\hline FN1 expression & & 0.12 \\
\hline Negative & 1 (Reference) & \\
\hline Positive & $1.68(0.88-3.19)$ & \\
\hline
\end{tabular}

${ }^{a}$ In the multivariate Cox regression analysis, FN1 expression was in the tumor cell cytoplasm was not retained as an independent predictor of tumor-specific survival. FN1, fibronectin 1; HR, hazard ratio. 
a role in clear cell RCC progression. It has been suggested that $\mathrm{FN}$ overexpression may be tumor-promoting in certain tumor types, such as PTC, hepatoblastoma (16) and ovarian cancer (17). Furthermore, a number of studies have indicated a significant role for FN in RCC. The first studies focusing on different RCC cell lines showed FN secretion in the culture medium and the possibility that $\mathrm{FN}$ affects the behavior of these malignant cells regarding movement and invasion (18-20). Murata et al showed that $\mathrm{FN}$ affected the migration of various RCC cell lines in culture. The SN12C-2 cell line with a high metastatic potential migrated most strongly when directed by FN gradient (18). In addition, Lohi et al showed the ability of various RCC cell lines to secrete different FN isoforms (19). The effect of ECM components, particularly FN, on renal tumor cell invasion was clearly demonstrated by Brenner et al (20). Wunderlich et al described a positive correlation of oncofetal FN expression with prognostic parameters and concluded that a high oncofetal FN may indicate poor outcome (21). Hegele et al supported this hypothesis but was unable to show any correlation between $\mathrm{cFN}$ plasma levels and nuclear grading (22). However, in our study we did not find a correlation between FN1 staining and patient age, gender, tumor differentiation and visceral metastasis. However, we found a trend for FN1 expression and correlation with tumor stage and lymph node metastasis. These findings indicate that there may be a correlation between $\mathrm{FN} 1$ protein expression and clinicopathological parameters, but due to several limitations in our study, such as the exclusion of other histological subtypes and limited number of cases as well as follow-up information, no significant correlation was noted. Furthermore, to the best of our knowledge, no studies have characterized the expression of FN1 in subcellular compartments (membranous vs. cytoplasmic). In addition, only a few studies have addressed the prognostic value of FN1 expression in clear cell RCC. Therefore, expertise in FN1 staining is lacking. An interesting study by $\mathrm{He}$ et al investigating the relationship of FN1 to von Hippel-Lindau gene inactivation, showed that in tumors with exon 1 and 2 mutations, FN had accumulated in the tumor cell cytoplasm and little or no FN was found in the stroma (23). However, in tumors without VHL mutations, FN was not present within tumor cells. These findings suggest that FN1 protein expression only plays a role in patients with VHL mutations.

In conclusion, to the best of our knowledge, this is the first study to show that FN1 protein expression in RCC cytoplasm is associated with a higher disease-related mortality rate, indicating a possible role in RCC progression. Therefore, our data on FN1 encourage further investigations, possibly with information about VHL mutation and a larger number of patients with follow-up to determine its precise role in renal cell cancer.

\section{References}

1. Schrader AJ, Sevinc S, Olbert PJ, Hegele A, Varga Z and Hofmann R: Gender-specific characteristics and survival of renal cell carcinoma. Der Urologe 47: 1184-1186, 2008.
2. Tamaskar I, Choueiri TK, Sercia L, Rini B, Bukowski R and Zhou M: Differential expression of caveolin-1 in renal neoplasms. Cancer 110: 776-782, 2007.

3. Wilson CB, Leopard J, Cheresh DA and Nakamura RM: Extracellular matrix and integrin composition of the normal bladder wall. World J Urol 14: S30-S37, 1996.

4. Akiyama SK, Olden K and Yamada KM: Fibronectin and integrins in invasion and metastasis. Cancer Metastasis Rev 14: $173-189,1995$.

5. Warawdekar UM, Zingde SM, Iyer KS, Jagannath P, Mehta AR and Mehta NG: Elevated levels and fragmented nature of cellular fibronectin in the plasma of gastrointestinal and head and neck cancer patients. Clin Chim Acta 372: 83-93, 2006.

6. Waalkes S, Atschekzei F, Kramer MW, et al: Fibronectin 1 mRNA expression correlates with advanced disease in renal cancer. BMC Cancer 10: 503, 2010.

7. Fuhrman SA, Lasky LC and Limas C: Prognostic significance of morphologic parameters in renal cell carcinoma. Am J Surg Pathol 6: 655-663, 1982

8. Stenzl A and De Kernion JB: Pathology, biology, and clinical staging of renal cell carcinoma. Semin Oncol 16 (Suppl 1): 3-11, 1989.

9. Theodorescu D, Broder SR, Boyd JC, Mills SE and Frierson HF Jr: Cathepsin D and chromogranin A as predictors of long term disease specific survival after radical prostatectomy for localized carcinoma of the prostate. Cancer 80: 2109-2119, 1997.

10. Merseburger AS, Hennenlotter J, Simon P, et al: Activation of the $\mathrm{PKB} / \mathrm{Akt}$ pathway in histological benign prostatic tissue adjacent to the primary malignant lesions. Oncol Rep 16: 79-83, 2006.

11. Merseburger AS, Kramer MW, Hennenlotter J, et al: Involvement of decreased Galectin-3 expression in the pathogenesis and progression of prostate cancer. Prostate 68: 72-77, 2008.

12. Tezval H, Jurk S, Atschekzei F, Serth J, Kuczyk MA and Merseburger AS: The involvement of altered corticotropin releasing factor receptor 2 expression in prostate cancer due to alteration of anti-angiogenic signaling pathways. Prostate 69: 443-448, 2009.

13. Peters I, Rehmet K, Wilke N, et al: RASSF1A promoter methylation and expression analysis in normal and neoplastic kidney indicates a role in early tumorigenesis. Mol Cancer 6: 49, 2007.

14. De la Fuente MT, Casanova B, Garcia-Gila M, Silva A and Garcia-Pardo A: Fibronectin interaction with alpha4beta1 integrin prevents apoptosis in B cell chronic lymphocytic leukemia: correlation with Bcl-2 and Bax. Leukemia 13: 266-274, 1999.

15. Danen EH and Yamada KM: Fibronectin, integrins, and growth control. J Cell Physiol 189: 1-13, 2001.

16. Takayasu H, Horie H, Hiyama E, et al: Frequent deletions and mutations of the beta-catenin gene are associated with overexpression of cyclin D1 and fibronectin and poorly differentiated histology in childhood hepatoblastoma. Clin Cancer Res 7: 901-908, 2001.

17. Tapper J, Kettunen E, El-Rifai W, Seppala M, Andersson LC and Knuutila S: Changes in gene expression during progression of ovarian carcinoma. Cancer Genet Cytogenet 128: 1-6, 2001.

18. Murata J, Saiki I, Yoneda J and Azuma I: Differences in chemotaxis to fibronectin in weakly and highly metastatic tumor cells. Jpn J Cancer Res 83: 1327-1333, 1992.

19. Lohi J, Tani T, Leivo I, et al: Expression of laminin in renal-cell carcinomas, renal-cell carcinoma cell lines and xenografts in nude mice. Int J Cancer 68: 364-371, 1996.

20. Brenner W, Gross S, Steinbach F, Horn S, Hohenfellner R and Thuroff JW: Differential inhibition of renal cancer cell invasion mediated by fibronectin, collagen IV and laminin. Cancer Lett 155: 199-205, 2000.

21. Wunderlich H, Wilhelm S, Reichelt O, Zermann DH, Borner R and Schubert J: Renal cell carcinoma in renal graft recipients and donors: incidence and consequence. Urol Int 67: 24-27, 2001.

22. Hegele A, Heidenreich A, Varga Z, et al: Cellular fibronectin in patients with transitional cell carcinoma of the bladder. Urol Res 30: 363-366, 2003.

23. He Z, Liu S, Guo M, Mao J and Hughson MD: Expression of fibronectin and HIF-lalpha in renal cell carcinomas: relationship to von Hippel-Lindau gene inactivation. Cancer Genet Cytogenet 152: 89-94, 2004. 